\title{
Quand les images citent l'art !
}

Anne Pauzet, UCO-Licia, Angers, CoDiRe, EA 4643, Université de Nantes, France

\section{Introduction}

Affiches, images publicitaires, dessins et photographies de presse... déclinent à l'infini les grandes images emblématiques de notre culture. Sous chaque image produite, on peut lire «"en transparence" une autre image et une autre formule cachées sous l'annonce "de surface" » (Fresnault-Deruelle 33). Intentionnel ou non, ce texte caché est un puissant révélateur culturel. La connaissance du soubassement rhétorique des messages visuels (Gauthier) permet alors de se distancier face à l'apparent réalisme des images (l'image comme image du monde) et de cheminer vers une reconnaissance de la dimension culturelle des messages visuels (l'image, reflet de notre représentation du monde)... De ces propos découlent autant de questions auxquelles il faudra répondre :

- Quels liens unissent images, art et inconscient collectif?

- De quelle façon ces messages iconiques se construisent-ils et se décodent-ils dans une culture donnée?

- Comment bâtir une véritable réflexion didactique pour l'enseignement et l'apprentissage des langues et des cultures?

- Quels sont les objectifs d'une telle démarche?

\section{Images et inconscient culturel : les images emblématiques}

Guy Gauthier explique que des images qui présentent extérieurement toutes les caractéristiques du réalisme - et en particulier des images photographiques exemptes de truquage - tirent leur sens de leur portée symbolique. Ce sont des images à valeur emblématique: «Nous parlons dans un monde, nous voyons dans un autre. L'image est symbolique mais elle n'a pas les propriétés sémantiques de la langue : c'est l'enfance du signe. Cette originalité lui donne une puissance de transmission sans égal. L'image fait du bien parce qu'elle fait lien. Mais sans communauté, pas de vitalité symbolique (...) » (Debray 60). Aussi, toute civilisation, parfois à son insu, vit d'emblèmes de ce genre, plus ou moins banalisés, plus ou moins sacralisés.

Dans son article «L'image et le savoir partagé : la Pietà de Benthalha » (1998), Pierre-Alban Delannoy s'interroge sur une photographie datant du 23 septembre 1997 à 
Bentalha, en Algérie. Cette photo, prise par un photographe de l'AFP, Hocine Zaourar, est aussi connue sous le titre «La Madone de Bentalha » et incarne les massacres des années 1990 en Algérie. Elle a littéralement fait le tour du monde et remporté le World Press Photo 1997 [lucarts.blogspot.com].

Une Piéta? Une Madone?

Pourquoi et comment est-ce ce sens-là qui s'impose d'emblée aux lecteurs occidentaux qui découvrent puis publient et popularisent ensuite, sous ces titres, cette photo ? C'est que la lecture d'une image s'appuie autant, sinon plus, sur un savoir préalable et partagé, et que lire l'image revient à transformer son contenu référentiel de manière à le faire entrer dans l'encyclopédie qui nous fait office de mémoire et de pensée. (Delannoy 56-57)

On y voit donc une femme en pleurs, la tête penchée. Une autre la console. De très nombreux clichés ont représenté cet évènement. Cependant c'est cette icône qu'a retenu l'Occident pétri de christianisme. Elle est la Madone car, pour les lecteurs, un certain nombre de traits additionnés en constituent le « type » (Groupe MU).

Les éléments gestuels, vestimentaires et plastiques, co-présents dans l'image, s'y transforment pour former l'archétype de la Pietà. Tout fait signe : les gestes et attitudes renvoient à la mère éplorée et à la douleur. Associés aux vêtements, ils rendent possible l'identification de cette femme en Pietà. La robe et le voile que portent les deux femmes, même s'ils sont en réalité des signes du mode de vie traditionnel du monde arabe, connotent, cependant, l'intemporalité. Dans ce contexte et de par ce cadrage, ils rendent possible l'identification de cette femme en Pietà car ils renvoient à l'époque du Christ ou bien au $15^{\mathrm{e}}$ siècle de Michel-Ange.

Mais cette image nous parle aussi, principalement, par sa facture :

Le cadrage, la position des personnages, la complémentarité des couleurs des deux robes (l'orangé et le bleu) et surtout la lumière. Celle-ci, en effet, donne au drapé des vêtements une matérialité, une densité sculpturale (...) qui ne peut manquer d'évoquer autant l'art de Michel-Ange sculpteur que celui des peintres qui ont excellé dans le rendu des plis des vêtements et en ont fait un trait plastique caractéristique de ces madones (...). (Delannoy 56-57)

Elle renvoie à toute une lignée d'images symbolisant la mort du Juste qui s'attachent à exprimer la douleur du Christ supplicié et des personnages qui l'entourent.

Comme l'a exprimé Pierre-Alban Delannoy, s'il avait manqué à cette photo la dimension plastique (composition et drapé), elle ne serait jamais apparue comme une image de la Madone. 
La mémoire collective est ainsi sollicitée par le recours à l'Art. Découvrir l'architecture secrète des images contemporaines, les codes invisibles du visible, renvoie donc aux grands archétypes qui ont trouvé leurs plus belles incarnations dans les œuvres des artistes: peintres, sculpteurs, photographes et cinéastes... La compréhension de cette rhétorique du visuel conduit à une vision plus large, plus approfondie des sociétés, de leurs valeurs, une approche de l'inconscient collectif qui en constitue le socle.

Comme l'écrit si joliment Alberto Manguel, les images ont pour vocation de transformer l'instant en éternité : « Mais l'histoire qu'elles recèlent demeure souvent cryptée et comme "illisible". A l'instar du baiser du prince réveillant quelque Belle au bois dormant, seul un regard peut alors réanimer ce qui a été ainsi “endormi” » (quatrième de couverture).

Entrer dans la culture de l'Autre par l'image nécessite donc de s'interroger sur les signifiés esthétiques auxquels elle renvoie. Dans un article fondateur en sémiologie de l'image, Roland Barthes met l'accent sur ce «signifié esthétique ». S'interrogeant sur la façon dont le sens vient à l'image et analysant une publicité Panzani, il exprime que l'on peut y voir une référence à la nature morte. Ce genre pictural se développe au dix-septième et dixhuitième siècle à une époque où les commanditaires des peintres cessent d'être uniquement la royauté. La bourgeoisie se développe et souhaite faire passer sa réussite sociale, son art de vivre à travers la peinture. L'allusion renvoie le lecteur à une certaine idée du bien-vivre : qualité des produits, fête des sens, plaisir de la table, de la bonne chair.

Cependant, si ce signifié esthétique semble souvent être utilisé par les concepteurs d'images, il n'est pas du tout évident que les récepteurs, et même parfois les concepteurs, puissent y faire explicitement référence. Pourtant il semble que nous décodons inconsciemment ce signifié esthétique comme s'il était stocké dans la vaste bibliothèque de références iconiques qui fait office de pensée.

A ces propos, nous ajouterons que

la variation des lectures n'est pas anarchique, elle dépend des différents savoirs investis dans l'image (savoir pratique, national, culturel, esthétique) et ces savoirs peuvent se classer, rejoindre une typologie ; tout se passe comme si l'image se donnait à lire à plusieurs hommes et ces hommes peuvent très bien coexister en un seul individu: une même lexie mobilise des lexiques différents. Qu'est-ce qu'un lexique ? C'est une portion du plan symbolique (du langage) qui correspond à un corps de pratique et de techniques; c'est bien le cas pour les différentes lectures de l'image. Chaque signe correspond à un corps d' «attitudes»: le tourisme, le ménage, la connaissance de l'art, dont certains peuvent manquer au niveau d'un individu. Il y a une pluralité et une coexistence des lexiques dans un même homme; le nombre et l'identité de ces lexiques forment en sorte l'idiolecte de chacun. (Barthes 48) 
Aussi est-il nécessaire de permettre aux apprenants et aux enseignants d'expliciter cet implicite visuel, de mettre au jour cette inter-iconicité de l'image afin que les messages deviennent de véritables monnaies d'échange interculturel, afin, aussi, de s'en distancer pour prendre du recul face à leur pouvoir de fascination...

Pour ce faire, il sera sans doute plus aisé, dans un premier temps, d'effectuer un travail à partir des images-citations les plus explicites : les «palimpsestes iconiques »(en référence aux palimpsestes verbaux-culturels évoqués par Robert Galisson).

\section{L’inter-iconicité : les palimpsestes iconiques}

Toute image est donc souvent la citation d'une image plus ancienne et l'inter-iconicité fonde l'existence de bien des images mass-médiatiques courantes (Fresnault-Deruelle).

Reconnaitre qu'une image est la citation d'une autre image éloigne le regard naïf face à la soit disant « objectivité » de l'image et l'idée qu'elle serait un pur reflet de la réalité.

Les messages iconiques jouent de cette inter-iconicité car il arrive bien souvent qu'une œuvre d'art, un genre artistique, un fragment d'œuvre ou encore l'artiste lui-même... concentrent à eux seuls la représentation que nous avons du monde.

L'art devient alors univers de référence et permet de mettre au jour une structuration spécifique à la culture étudiée. Le phénomène existe ici et ailleurs et se complexifie à mesure de l'évolution de la rhétorique des images et de notre capacité à les décoder. Il peut aussi bien servir la propagande politique que véhiculer des messages spirituels, sociaux ou bien, plus prosaïquement, commerciaux... On recourt à la richesse symbolique des œuvres artistiques pour apporter une valeur ajoutée aux messages. L'inconscient de la création iconique apparait au grand jour, nourri de mythes, d'archétypes ou de religion... C'est dans le but explicite de solliciter la mémoire collective, de créer des univers représentant visuellement les valeurs dont ils désirent que leurs images soient porteuses que les concepteurs se mettent à puiser dans les vastes réserves du patrimoine.

Pour exemple, entre 1962 et 1973, la Chine de Mao a largement usé de ce procédé : « une signalétique révolutionnaire très précise, greffée sur des figures symboliques issues de la tradition, était destinée à agir sur la conscience et l'inconscient collectifs » (collection Gorsky). Ainsi la tradition, si violemment décriée par ailleurs, servait les besoins de la propagande. Des peintures traditionnelles, détournées et reproduites à des millions d'exemplaires, glorifiaient les progrès et valeurs du socialisme : montagnes élancées peintes 
d'un trait de pinceau à l'encre de Chine auxquelles les peintres se devaient d'ajouter de fiers pylônes électriques, quelques drapeaux rouges ou des vélos bien prolétaires... Ou encore,

la représentation de quelques thèmes chers au cœur des Chinois, tels les canards s'ébrouant dans l'eau, [devait] répondre aux critères de la nouvelle société. Alors que, autrefois, on représentait toujours une couple de canards mandarins en faisant clairement ressortir les couleurs chatoyantes du mâle et le plumage discret de la femelle, et que chacun y voyait clairement une allusion au bonheur conjugal, la mare aux canards glorifiait désormais les joies de la vie commune, avec un troupeau compact et uniforme, dans lequel il serait bien difficile de distinguer le canard et sa canette. (Collection Gorsky VIII)

Autre exemple, venu d'Allemagne cette fois : une affiche de Klaus Staeck [(sous la direction d'Alain Weill), L'envers de la porte de Brandebourg (1990)]. Derrière la porte de Brandebourg, à Berlin, l'auteur de cette affiche a installé une image emblématique de la culture allemande, le tableau de Caspar David Friedrich, La Mer de glace, dont le titre devint par la suite L'Espoir échoué. La citation à l'identique de cette œuvre renvoie à la difficile réunification des deux Allemagnes, les Allemands de l'Est déplorant le froid social des Allemands de l'Ouest.

Les concepteurs d'images sollicitent donc la mémoire collective en puisant dans les œuvres artistiques du patrimoine collectif. Ces messages sont intentionnels. Ressentis et vécu dans un premier temps, ils peuvent ensuite faire l'objet d'une véritable enquête, un travail d'élaboration du sens. Ces références visuelles peuvent être nommées et classées et renvoyer au style d'une époque, d'un artiste (« A la manière de... ») (Fava), une œuvre particulière, un genre, à l'artiste lui-même, à un support artistique ou aux codes en vigueur dans le domaine artistique.

Quand les images citent l'Art, l'apprenant peut tout à la fois découvrir la culture quotidienne véhiculée par les médias mais accéder aussi à une culture plus artistique, plus symbolique et savante dans un va et vient allant de l'image source (l'œuvre d'art) à l'image détournée. Si les images sont fruits d'une culture et reflètent la représentation que nous avons du monde, elles constituent alors un bon terrain de réflexion pour les apprenants de langue.

Cependant, entrer dans l'image, c'est aussi s'immerger dans un monde de forme et de couleur qui parle tout d'abord à notre corps, c'est entrer dans un monde sensible... viennent ensuite l'envie de comprendre et d'en savoir plus. Ces deux dimensions, raison et sensibilité, ne doivent pas s'opposer comme on a le fait si souvent mais se compléter. 
Le monde de l'œuvre est une unité $[\ldots]$ mais $[\ldots]$ au sein de cette totalité, il est possible d'opérer des distinctions «le sensible, l'objet représenté, le monde exprimé » qui s'adressent à trois instances du sujet : au corps par le langage des formes qui font naitre des sensations, à l'intelligence en appelant des références qui construisent des significations, au sentiment enfin en provoquant des engagements du spectateur. (Richard 162-163)

Ceci nous conduit à une démarche de réception : «Partant de l'œuvre, de sa capacité à se rendre sensible aux mouvements qu'elle construit, le spectateur laisse remonter en lui les interprétations, puis va chercher à les contrôler » (162 -163).

Par empathie, nous partageons le monde de l'Autre, cet Autre qui peut ensuite verbaliser les réseaux souterrains de significations de l'image afin qu'elle devienne une véritable monnaie d'échange interculturelle pour peu que les apprenants disposent d'outils permettant d'en révéler la charpente secrète et les représentations qui y sont associées.

Déambuler au fil de l'inter-iconicité des images, partager, comprendre et pourquoi pas créer ensemble de nouvelles images, telles sont les voies didactiques à privilégier. L'Art, comme univers de référence, nous ouvre alors des chemins de découverte, une vision profonde, humaniste pour la compréhension de l'autre et la rencontre.

\section{Applications didactiques et objectifs : les « passeurs de culture »}

Voici deux exemples de travaux réalisés depuis quelques années avec des étudiants étrangers ou français. Ils sont l'aboutissement concret des propos théoriques cités précédemment.

\section{A. Palimpsestes iconiques}

Depuis de nombreuses années, le CIDEF (Centre International d'Études Françaises, Université Catholique de l'Ouest, Angers) propose aux étudiants un cours optionnel intitulé «Images et cultures » (trois heures par semaine sur un semestre). Ce cours a été mis en place à mon initiative et articule recherche, interculturel et enseignement-apprentissage des langues. Il s'adresse à des groupes d'étudiants adultes de niveau B2/C1. Une partie du cours porte sur les images emblématiques et les «palimpsestes iconiques ».

Le cours commence par la présentation d'un power-point citant quelques palimpsestes glanés dans les journaux et les magazines français. Pour chacun de ces palimpsestes, les apprenants doivent répondre, par groupes multiculturels, aux questions suivantes :

- Regardez les images proposées. Quelles sont vos premières impressions ?

- A quelles œuvres vous font-elles penser? 
- Comparez chaque image à l'œuvre originale. Que remarquez-vous ? Quelles sont les différences? Qu'est-ce qui a été réutilisé (forme, couleurs...) ? Qu'est-ce qui a été modifié ?

- Pourquoi a-t-on modifié les images de cette façon-là ?

Un premier échange d'impressions, de points de vue, de remarques, d'hypothèses a alors lieu. C'est de la confrontation des lectures que naitra le recul, acquis important pour fonder une attitude distanciée et raisonnée face à l'image et au monde qu'elle reflète (Hamm, 1986).

Les étudiants sont ensuite invités à faire une recherche plus détaillée en complétant deux fiches d'étude : une pour l'œuvre originale, une pour le palimpseste.

Fiche d'étude de l'œuvre originale (inspirée de Galisson) : Nom de l'artiste, titre de l'œuvre, date de l'œuvre, technique, lieu de conservation (...), contexte de parution, présentation de l'artiste, informations sur l'œuvre (on retiendra surtout les informations qui pourront permettre une compréhension plus fine du palimpseste).

Fiche d'étude du palimpseste iconique (inspirée de Galisson): Présentation de l'auteur (dans le cas d'une publicité, présentation de l'annonceur), support, cible ou public visé, date de parution, type de détournement (citation intégrale de l'œuvre, fragment d'œuvre, œuvre «à la manière de », évocation culturelle), écarts observés, effets produits / effets de sens.

Chaque groupe présente un palimpseste et l'enseignant ajoute éventuellement des informations, produit d'autres documents, complète, montre en quoi elle est emblématique d'une époque, d'une représentation du monde. Il ouvre de nouvelles pistes de réflexions...

Dans cette démarche la langue française n'est plus une fin en soi mais un moyen d'accéder à l'image et ses réseaux de significations.

Sur le même modèle, les apprenants seront ultérieurement invités à créer eux-mêmes un palimpseste, à jouer avec les œuvres, les couleurs, les formes pour illustrer ou promouvoir une idée, un produit de leur choix. Chaque groupe présente son palimpseste et les choix qui ont présidé à son élaboration.

La création collective initie à la rhétorique de l'image de façon ludique, motive par le recours à la force émotionnelle et la richesse des symboles et par la sollicitation de l'inconscient personnel et collectif. On partage, par empathie, la création de l'autre. 
Suite à ce travail, les étudiants peuvent présenter un palimpseste issu de la culture de leur choix. Chacun sera libre de présenter l'image qu'il souhaite car c'est un facteur de motivation. L'univers choisi sera propre à chacun et lui permettra de s'impliquer davantage.

\section{B. La « traduction » culturelle d'images mass-médiatiques courantes}

Dans un tout autre contexte, il m'a été demandé de mener un cours d'une dizaine d'heures à l'IPLV (Institut Pour les Langues Vivantes, Université Catholique de l'Ouest à Angers). Ce cours, intitulé MIEL (Message Iconique et Environnement International), s'adresse à des étudiants en master 1 LACISE (Langue, Communication Interculturelle et Stratégie d'Entreprise). Ce cours constitue une sensibilisation aux questions suivantes :

- En quoi la lecture de l'image est-elle culturelle ?

- Quels sont les outils permettant de procéder à une adaptation culturelle d'images massmédiatiques courantes?

Un module présente plus particulièrement le "signifié esthétique » cher à Roland Barthes et évoque l'inter-iconicité des images par le biais des palimpsestes iconiques.

B1. Réalisation d'un dossier : adaptation culturelle d'une image fixe

L'image nous raconte une histoire en une apparition qui doit tout dire! Aussi les étudiants sont-ils invités à trouver une publicité ou une affiche pour un produit (ou une idée). Cette campagne "publicitaire » doit déjà exister en France et jouer sur des représentations locales. L'étudiant doit alors imaginer une adaptation de cette campagne pour l'étranger. Ils devront choisir sous quelle forme adapter cette campagne. Créer un "palimpseste iconique » peut être une option possible en fonction du message qu'ils veulent faire passer.

B2 : Exemple

Le dossier d'une d'étudiante (Devaux) illustre bien ces possibles emprunts à l'Art. Il joue sur la référence à un modèle esthétique qui a forgé la perception des espagnols.

Cette étudiante a choisi d'adapter l'huile d'olive Puget sur le marché espagnol. Alors qu'en France l'huile d'olive est souvent associée au Sud et à son «folklore » (le soleil, les cigales, l'accent et les oliviers...), il lui a fallu substituer un imaginaire à un autre. Pour les Espagnols, en effet, ce produit a de tout autres connotations. Les Espagnols en parlent et le goutent comme un vin : son gout dépend de la terre, de la variété d'olives, du pourcentage d'acidité...

Plus qu'une huile, c'est un produit presque sacré. Aussi l'étudiante a-t-elle eu l'idée d'adapter la nouvelle publicité sous la forme d'un « Bodegone ». Au $17^{\mathrm{e}}$ et $18^{\mathrm{e}}$ siècles, l'âge d'or des plus brillants peintres espagnols (Velasquez, Goya...), les artistes peignent ces 
natures mortes. Les «Bodegones » renvoient «au sacré » et «à la simplicité » et dégagent une impression de rigueur sans rigidité et d'ascétisme sans sévérité.

L'allusion au Bodegone a donc permis de véhiculer le message suivant : « Les choses simples, de tous les jours, sont sacrées. L'huile d'olive est un produit simple, pour tous les jours mais sacré » (Devaux, np). Modernisé, ce Bodegone a ensuite connoté un autre réseau de significations : « le luxe », « la magnificence des produits » et leur « pureté ».

\section{Conclusion}

Convaincue qu'une éducation à l'Art est une bonne propédeutique à une culture du visuel quel qu'il soit, je fais le souhait que ce travail, tout en dotant les lecteurs et apprenants d'outils de réflexion, puisse leur permettre d'aborder les rivages de ces I.L.E.S (Images Langues Étrangères) (Lambert) avec curiosité.

Apprendre par l'Art, dans un lieu où les images issues des réalités étrangères foisonnent, m'a toujours semblé une utopie souhaitable pour apprendre la rencontre.

Chacun y mettra un peu de lui-même, se dévoilera tout en restant protégé, ce qui est le propre de la médiation d'une parole par le truchement de l'Art (Clerc).

Chacun deviendra un peu «passeur de culture ».

\section{Bibliographie}

Barthes, Roland. « Rhétorique de l'image ». Communications 4 (1964) : 40-51.

Clerc, Stéphanie. «Récits, écriture et mise en scène en classe d'accueil ». Les Langues Modernes. Pratiques artistiques et pratiques langagières : quelle synergie? Revue de l'APLV 2 (2010) : 65-72.

Debray, Régis. Vie et mort de l'image. Paris : Folio Essais, 1992.

Delannoy, Pierre-Alban. «L'image et le savoir partagé ». Communication et langages 115 (1998) : 52-58.

Devaux, Elise. «Messages iconiques et environnement international: adaptation de la publicité pour l'huile d'olive de la marque Puget pour l'Espagne ». Dossier de Master 1 Lacise, UCO, Angers, 2010 (non publié).

Fava, Véronique. "“A la manière de": l'œuvre d'art dans l'affiche commerciale ». Communication et langages 95 (1993) : 55-66.

Fresnault-Deruelle, Pierre. L'image manipulée. Paris : Médiathèque, Edilig, 1983.

Galisson, Robert. «Les palimpsestes verbaux : des révélateurs culturels remarquables, mais peu remarqués... ». Les cahiers de l'ASDIFLE 6 (1995) : 41-64. 
Gauthier, Guy. Vingt + une leçons sur l'image et le sens. Paris : Médiathèque, Edilig, 1982.

Gorsky, Claude. La Chine à l'affiche (collection d'affiches : préface de Zoé Valdès). Paris : Ramsay, 1997.

Groupe Mu. Traité du signe visuel. Paris : Seuil, 1990.

Hamm, Liliane. Lire des images. Paris : Armand-Colin Bourrelier, 1986.

Lambert, Frédéric. «Images Langues Étrangères ». Le Français dans le Monde. Numéro spécial (juillet 1994) : 35-38.

Manguel, Alberto. Le livre d'images. Tr. française. Paris : Actes Sud, 2001.

Richard, Annette. «Itinéraires pour une appropriation des œuvres picturales. (Finalités et conditions d'une initiation à l'art chez des élèves de lycée. Propositions d'une méthode)». Thèse de doctorat (Nouveau Régime), Université Paris VIII : UFR Esthétiques, Sciences et Technologies des Arts. Théories et Pratiques des Arts Plastiques, 1994.

Weill, Alain, dir. Affiches politiques et sociales. Sixième rencontres internationales des arts graphiques. Paris : Somogy / Éd. d'Art, 1995. 